\title{
Long non-coding RNA TUC338 is functionally involved in sorafenib-sensitized hepatocarcinoma cells by targeting RASAL1
}

\author{
WEIDONG JIN $^{1 *}$, LEI CHEN $^{2 *}$, XUN CAI $^{1}$, YUNXIAO ZHANG $^{3}$, JIANXIN ZHANG $^{1}$, \\ DANGDANG MA ${ }^{1}, \mathrm{XIONG} \mathrm{CAI}^{1}$, TAO FU ${ }^{1}$, ZHENGPING YU ${ }^{4}$, FUXIANG YU ${ }^{4}$ and GANG CHEN ${ }^{4}$ \\ ${ }^{1}$ Department of General Surgery, Wuhan General Hospital of Guangzhou Military, Wuhan, Hubei 430000; \\ ${ }^{2}$ Department of Hepatobiliary Surgery, Wenzhou Central Hospital; ${ }^{3}$ Renji College of Wenzhou \\ Medical University; ${ }^{4}$ Department of Hepatobiliary Surgery, The First Affiliated Hospital, \\ Wenzhou Medical University, Wenzhou, Zhejiang 325000, P.R. China
}

Received May 29, 2016; Accepted October 25, 2016

DOI: $10.3892 / o r .2016 .5248$

\begin{abstract}
Development of novel targeted therapy holds promise for conquering chemotherapy resistance, one of major hurdles in current liver cancer treatment. We found that long non-coding RNA TUC338 is involved in the development of hepatocellular carcinoma (HCC) and sorafenib resistance. HCC cell lines were transfected with siTUC338, then cell proliferation and invasion ability were investigated by MTT and Transwell assay. Sorafenib resistance HepG2 cells were generated to test the role of TUC338 in sorafenib sensitivity. Intratumoral delivering of siTUC338 was used to analyze the sorafenib treatment response in HepG2/Sor xenografts in vivo. Higher levels of TUC338 were found both in HCC tissues and cell lines, knockdown of TUC338 was accompanied with increased expression of RASAL1 in HCC cell line with increased proliferation and invasion ability, knockdown of TUC338 could activate the RASAL1 pathway and inhibit tumor growth genes by directly targeting RASAL1 3'-UTR. Furthermore, knockdown of TUC338 in HepG2 sorafenib sensitized its reaction to the treatment of sorafenib, which was accompanied by increased expression RASAL1; intratumoral delivering of siTUC338 could also restore sorafenib treatment response in HepG2/Sor xenografts in vivo. These findings provide direct evidence that the TUC338/RASAL1 axis might play an essential role in sorafenib-resistance of liver cancer cells, suggesting the signaling cohort could serve as a novel
\end{abstract}

Correspondence to: Dr Gang Chen or Dr Fuxiang Yu, Department of Hepatobiliary Surgery, The First Affiliated Hospital, Wenzhou Medical University, Wenzhou, Zhejiang 325000, P.R. China

E-mail: gangchen1982@163.com

E-mail: yfxbmu@163.com

*Contributed equally

Key words: TUC338, RAS protein activator like-1, liver cancer, chemotherapy resistant, sorafenib therapeutic target for the treatment of chemotherapy resistant liver cancer.

\section{Introduction}

Hepatocellular carcinoma (HCC) is the most common primary liver tumor and the third most frequent cause of cancerrelated death worldwide (1). Surgical resection and traditional chemotherapy are typical forms of treatment for patients with HCC (2). Sorafenib (Nexavar) is the first and only targeted therapy to clinically improve overall survival in patients with advanced $\mathrm{HCC}$, and has given hope to researchers for effective agents to combat $\mathrm{HCC}$ (3), but the clinical response is seriously limited by drug resistance. To develop optimum strategy for overcoming sorafenib treatment resistance is one of the major concerns in currently liver cancer treatment. Long-lasting endeavors have been made in identifying genes inducing chemoresistances in the past decades $(4,5)$. However, the link between specific gene and sorafenib treatment resistance in liver cancer therapy remains unclear.

Non-coding RNAs (ncRNA) have recently been implicated in hepatocarcinogenesis and tumor progression, represent promising targets for cancer (6), ncRNAs include short and long non-coding RNAs (7). Short ncRNAs have a length of under 200 nucleotides (nt) while long ncRNAs (lncRNAs) are more than $200 \mathrm{nt}$ in length, frequently ranging up to $100 \mathrm{~kb}(8)$. Recent studies estimated that the number of lncRNAs in humans is approximately 15,000 and found that most lncRNAs displayed tissue-specific expression patterns (9). Increasing evidence identified that 1 chNAs epigenetically regulate the expression of genes involved in fundamental cellular processes such as cell proliferation, apoptosis, differentiation, and migration, suggesting their potential oncogenic or tumor suppressing roles in cancer development (10). The TUC338 (transcribed ultra-conserved region 338) gene was first identified as uc.338, along with 480 other ultra-conserved elements in the human genome. Expression of this RNA gene has been found to markedly increase in hepatocellular carcinoma (HCC) cells (11). Moreover, TUC338 expression correlates with disease progression, and raises the possibility that it may be involved in malignant transformation (12). It is also 
reported that lost restoration of TUC338 in liver cancer cells inhibit their cell proliferation and invasion (13), indicating a functional role in liver cancer cell growth.

RAS proteins control many cellular processes, including cell migration, proliferation, differentiation, and survival. The RAS GTPase-activationg protein (RasGAP) gene RASAL1 has been demonstrated as a tumor suppressor gene which act as a negative modulator of the RAS signaling pathway by catalyzing RAS inactivation (14). RASAL1 expression is decreased in many tumors, including colorectal tumor (15), thyroid cancer (16), gastric cancer (17), prostatic cancer and bladder cancer (18). However, no data have been reported on its association with chemoresistance. RASAL1 is stimulated by increases in intracellular $\mathrm{Ca}^{2+}$ leading to the attenuation of RAS activation and MAPK activity, contributing to tumor progression through its weakened anti-RAS activity (19). Activated RAS is involved not only in tumor progression but also possibly in the development of resistance of tumor cells to chemotherapy and to ionizing radiation (20), RASAL1 might inhibit tumor development and sensitivity to drug-therapy by negatively regulating RAS activation.

Based on findings that the TUC338 and RASAL1 functionally regulate the tumor progression, we postulated that TUC338 might play a role in the chemotherapy resistance of liver cancer cells, which might be mediated by its regulation of the expression of RASAL1. In the present study, we explored the functional significance of TUC338/RASAL1 axis in the sorafenib-resistance in liver cancer cells by in vitro and in vivo studies.

Our results showed that elevated expression of TUC338 was found in HCC tissues and cell lines, and knockdown TUC338 in liver cancer cells inhibit the cell proliferation and invasion ability accompanied with the overexpression of RASAL1. Furthermore, knockdown TUC338 inhibited the RASAL1 pathway and decreased the tumor growth genes, by increase the RASAL1 transcription. Silencing of TUC338 in HepG2/Sor cells could also decrease the cell proliferation and eradicated its resistance to sorafenib in vitro and in vivo. These findings suggest that TUC338 might promote sorafenibresistance in liver cancer, which is mediated via its regulation of RASAL1 and this might be a novel therapeutic target for sorafenib-resistant liver cancer therapy.

\section{Materials and methods}

Samples. Twelve pairs of HCC samples and adjacent non-tumor tissues were obtained from surgical specimens at department of hepatobiliary surgery, the first affiliated hospital of Wenzhou Medical University (Wenzhou, China) after informed consent. Clear hepatocellular carcinoma was diagnosed histopathologically. All these specimens were snap-frozen in liquid nitrogen after excision. The study methodologies conformed to the standards set by the Declaration of Helsinki. Informed consent was obtained from each participant, collection and usage of all specimens were approved by the local ethics committee.

Cell culture and reagents. The human hepatoma cell lines, HepG2, SMMC-7721, BEK-7402, Hep3B, and Huh-7, and the liver cell line L02 were purchased from the cell bank of Type Culture Collection at the Chinese Academy of Sciences
(Shanghai, China). The cells were maintained in RPMI-1640 or DMEM supplemented with $10 \%$ fetal bovine serum (FBS), $100 \mathrm{U} / \mathrm{ml}$ penicillin and $100 \mu \mathrm{g} / \mathrm{ml}$ streptomycin at $37^{\circ} \mathrm{C}$ in $5 \% \mathrm{CO}_{2}$ incubation. Sorafenib (BAY 43-9006) was purchased from MedChem Express (Princeton, NJ, USA) and dissolved in DMSO. The final DMSO concentration was $<0.1 \%$.

siRNA and transfection. For TUC338 knockdown experiments, siRNA was designed and synthesized by GenePharma Co., Ltd (Shanghai, China), and the sequences were as follows: si-TUC338: SenseSeq: 5'-CCACAGGACAGGUACAGC ATT-3', AntiSeq: 5'-UCAGUCUCCAGGGCUGUCATT-3'; si-NC: SenseSeq: 5'-UUCUCCGAACGUGUACGUTT-3', AntiSeq: 5'-ACGUGACACGUUCGGAGAATT-3'. HepG2, SMMC-7721 and Huh-7 cells were plated at a density of $2 \times 10^{5}$ cells/well in 6-well plates, overnight culture, cells were transfected with si-NC or si-TUC338 by Lipo2000 (11668019, Life Technology) using $100 \mathrm{nM} /$ well siRNA in a 6-well plate following the manufacturer's instructions. At $48 \mathrm{~h}$ after transfection, total protein and RNA samples were prepared, proliferation assay, invasion assay, luciferase assay were carried as described below.

Real-time PCR. Total RNA was extracted using TRIzol ${ }^{\circledR}$ reagent (Invitrogen). DNaseI-treated RNA was used for first strand cDNA synthesis using M-MLV reverse transcriptase (Promega) and oligo (dT) 15 according to the manufacturer's protocols and $1 \mu \mathrm{l}$ cDNA samples were used for conventional PCR amplification. Real-time quantitative PCR analysis was performed in a real-time PCR system (StepOne, Applied Biosystems) and the expression levels of TUC338 were normalized to $18 \mathrm{~s}$ determined by a SYBR Green-based comparative cycle threshold CT method. Real-time PCR primers were: TUC338-F: 5'-GGTGAGAGGGGATGT TCAGT-3', TUC338-R: 5'-TGGGTGAAATGAGGTTG GGG-3'; RASAL1-F: 5'-TGGATTTCTCTTCTTGCGAT TCT-3', RASAL1-R: 5'-TGTTGGTCCCGAAGGTCAA-3'; VEGF-F: 5'-AGGGCAGAATCATCACGAAGT-3', VEGF-R: 5'-AGGGTCTCGATTGGATGGCA-3'; GLUT-1-F: 5'-GGC CAAGAGTGTGCTAAAGAA-3', GLUT-1-R: 5'-ACAGCGT TGATGCCAGACAG-3'; MDR-1-F: 5'-TTGCTGCTTACA TTCAGGTTTCA-3', MDR-1-R: 5'-AGCCTATCTCCTGTC GCATTA-3'; 18s-F: 5'-CGCTTCCTTACCTGGTTGAT-3', 18s-R: 5'-GAGCGACCAAAGGAACGATA-3'.

Western blotting. Total proteins were extracted with RIPA buffer containing proteinase/phosphatase inhibitors (Thermo Fisher Scientific, Cambridge, MA, USA). Thirty micrograms of cell lysate and tumor tissue lysate were separated on $12 \%$ sodium dodecyl sulfate-polyacrylamide gel electrophoresis (SDS-PAGE) gels and then transferred to Immobilon-P transfer membrane (Millipore). Specific anti-RASAL1 (ab170711, 1:1000 dilution), anti-VEGF (ab1316, 1:1000 dilution), antiGLUT-1 (ab115730, 1:1000 dilution), anti-P-glycoprotein (ab170904, 1:1000 dilution), anti- $\beta$-actin (ab3280, 1:3000 dilution) primary antibodies (Abcam, USA) were used, and HRP conjugated immunoglobulin was used as a secondary antibody (Zen BioScience, Chengdu, China). Immunoblot analysis was performed with the indicated antibodies and visualized with Immobilon Western (Millipore), which were quantified using 


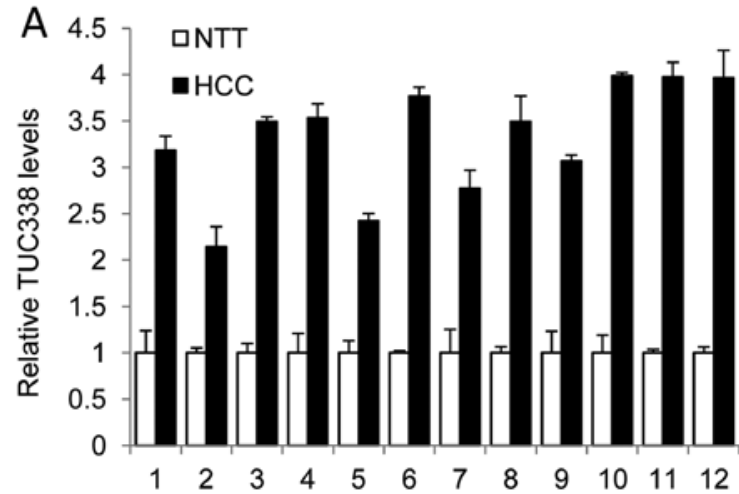

B

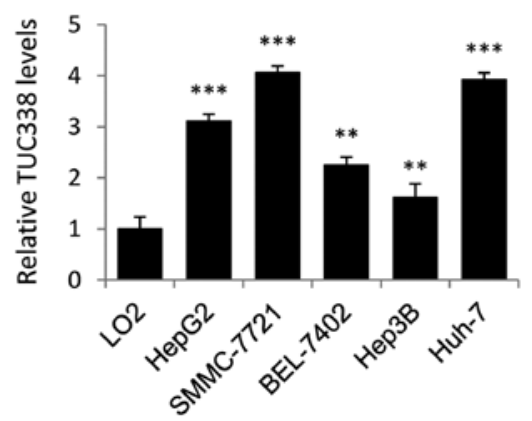

Figure 1. Upregulation of TUC338 in human hepatocarcinoma (HCC) tissues and cell lines. (A) Real-time PCR analysis of TUC338 expression in 12 HCC specimens compared to their pair-matched adjacent non-tumor tissues (NTT). Expression levels were normalized to $18 \mathrm{~s}$ and expressed as relative change compared to NTT. (B) Real-time PCR analysis of TUC338 expression in the liver cell line L02 and HCC cell lines HepG2, SMMC-7721, BEL-7402, Hep3B, and Huh-7. Expression levels were normalized to $18 \mathrm{~s}$ and expressed as relative change compared to L02. Data are shown as mean \pm SEM of three independent experiments. The results were reproduced in three independent experiments. ${ }^{* *} \mathrm{P}<0.01,{ }^{* * *} \mathrm{P}<0.001$ compared to $\mathrm{LO} 2$ group. Data are shown as mean $\pm \mathrm{SEM}$ of three independent experiments.

densitometry image analysis software (Image Master VDS; Pharmacia Biotech Inc.). Normalization was made against $\beta$-actin expression.

In vitro cell growth assays

Cell counting. Viable cells were counted as previously described (21). When the cells were cultured to $70-80 \%$ confluence, sorafenib was added into the medium for different concentrations at different time periods. All counts were performed on triplicate wells and repeated in three independent experiments and mean \pm SEM of cell number was plotted against culture duration of 8 days. For the sorafenib treatment assay, the cell amount was confirmed to be comparable in the starting day for each cell line by adjusting the cell amounts seeded according to their doubling time.

MTT assay. Cells were seeded at $5 \times 10^{3}$ cells/well and cultured under various concentration of sorafenib for different time periods. Viable cells were determined by MTT assay as previously described (22).

In vivo tumor study. Male nude mice (4-6 weeks, 18-20 g) were purchased from the Model Animal Research Center of Nanjing University. The animals were housed in a temperature- and humidity-controlled room with a 12-h on-off light cycle and given free access to food and water. To establish the exnograft mouse model, HepG2/Sor sorafenib-resistant cancer cells were diluted with phosphate-buffer saline (PBS) at a concentration of $2 \times 10^{7}$ cells $/ \mathrm{ml}$, and $200 \mu \mathrm{l}$ HepG2/Sor cells were injected subcutaneously into the left back limb of each mouse. Tumor volumes were determined every five days after injection and calculated as previously described (23). Mice with xenograft volume $>500 \mathrm{~mm}^{3}$ were treated with $4 \mathrm{mg} / \mathrm{kg}$ sorafenib (Sor) intraperitoneally (i.p.) combined with intratumoral injection of physiological saline twice a week (Sor+saline group), siNC (Sor+siNC group) and siTUC338 (Sor+siTUC338 group) for four weeks. Mice were sacrificed and tumors were dissected for real-time PCR and western blot analysis. The animal study was carried out in strict accordance with the recommendations in the guide for the care and use of laboratory animals of the First Affiliated Hospital of Wenzhou Medical University and Research Institute Animal Care and Use Committee. All the protocols were approved by the First Affiliated Hospital of Wenzhou Medical University and Research Institute Animal Care and Use Committee (approval number 21040608). Surgery was performed under sodium pentobarbital anesthesia, and all efforts were made to minimize suffering.

Plasmid construction and luciferase assay. To determine whether the entire human RASAL1 3'-untranslated region (UTR) segment was amplified by PCR homo-genomic DNA was used as a template. The PCR products were inserted into the pGL3-Basic plasmid (Ambion). For luciferase reporter assays, $1 \mu \mathrm{g}$ of firefly luciferase reporter plasmid, $0.5 \mu \mathrm{g}$ of $\beta$-galactosidase expression vector (Ambion), and equal amounts $(200 \mathrm{pmol})$ of siNC or siTUC338 were transfected into cells in 6-well plates. The $\beta$-galactosidase vector was used as a transfection control. At $48 \mathrm{~h}$ after transfection, cells were assayed using luciferase assay kits (Promega).

Statistical analysis. The results are expressed as mean \pm SEM from at least three independent experiments. Significance analysis of normally distributed data were performed using two-tail Student's t-test and P-values of $<0.05$ and 0.01 were considered significant and highly significant, respectively.

\section{Results}

TUC338 expression is significantly increased in HCC tissues and cell lines. To determine whether TUC338 was involved in regulation of human $\mathrm{HCC}$ tumorigenesis, we first detected TUC338 levels in HCC tumor and adjacent non-tumor tissues, using real-time PCR $(n=12)$. As shown in Fig. 1A, TUC338 expression was significantly upregulated in HCC samples compared to normal adjacent liver tissue. We also analyzed TUC338 expression in liver cell line L02 and five human HCC cell lines (HepG2, SMMC-7721, BEK-7402, Hep3B, and Huh-7). Consistently, TUC338 was increased in all HCC cell lines examined compared to L02 cells (Fig. 1B). Taken together, these findings suggested that TUC338 is upregulated in human HCC.

Inhibition of TUC338 decreases cell proliferation and invasion by activation of RASAL1. Functional role of TUC338 in liver cancer cells was explored by siRNA knockdown TUC338 
A
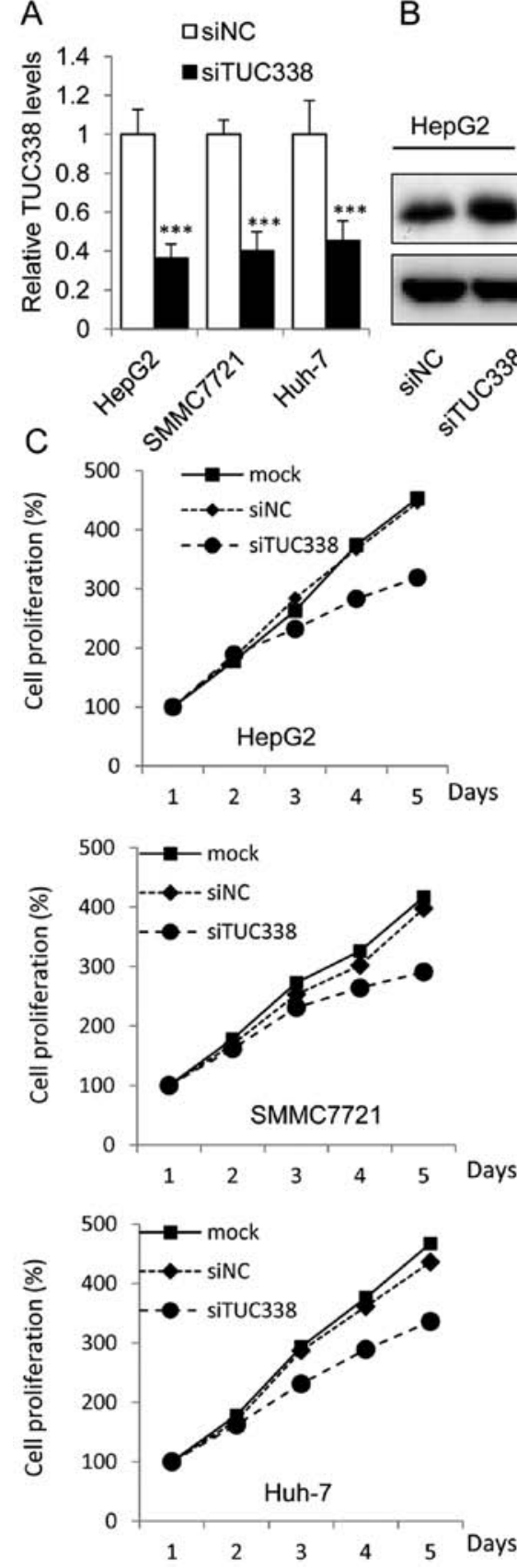

B

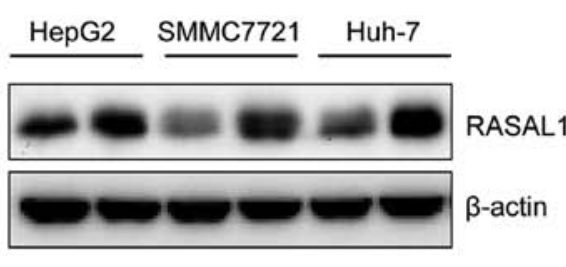

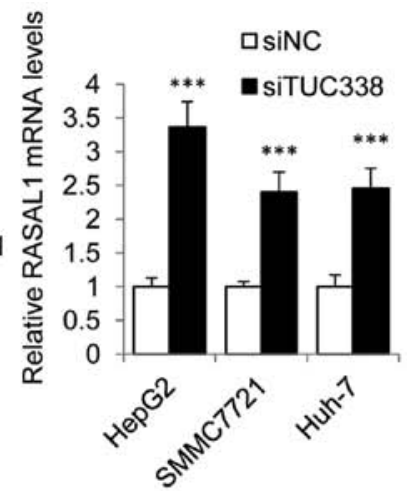

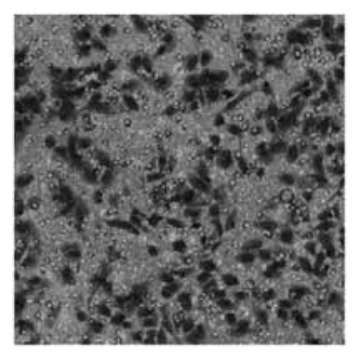

siNC
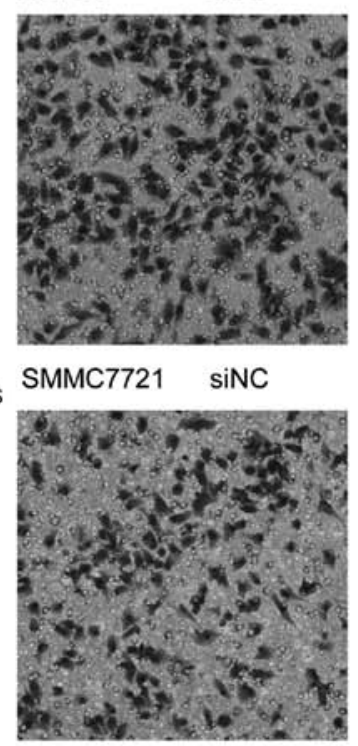

Huh-7

siNC

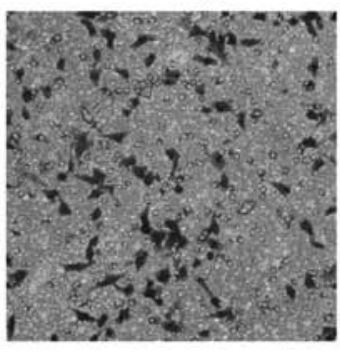

siTUC338

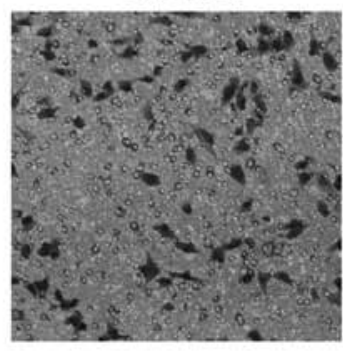

siTUC338

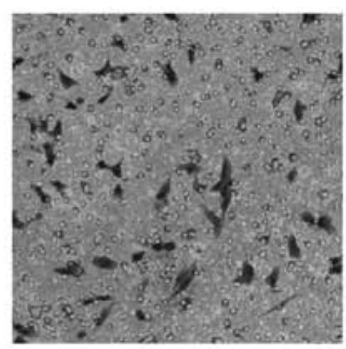

siTUC338

Figure 2. Knockdown of TUC338 inhibits cell proliferation and invasion by increasing the expression of RASAL1. (A and B) TUC338 level was detected by real-time PCR, RASAL1 protein and mRNA levels were determined by western blot and real-time PCR at $48 \mathrm{~h}$ after transfection with siNC or siTUC338, $\beta$-actin was used as loading control. (C) Cumulative cell growth curve expressed by MTT assay in HepG2, SMMC7721 and Huh-7 cells trasfected with siNC or siTUC338 (left panel). Cell invasion ability was demonstrated using Transwell assay (right panel). Each bar represents the mean \pm SEM. The results were reproduced in three independent experiments. ${ }^{* * *} \mathrm{P}<0.001$ compared to siNC group. Data are shown as mean $\pm \mathrm{SEM}$ of three independent experiments.

in HepG2, SMMC7721 and Huh-7 cells which showed higher basal expression levels of TUC338. It was observed that the expression of TUC338 was significantly downregulated $48 \mathrm{~h}$ after transfection with siTUC338 by Lipo2000 in mRNA levels as compared with the siNC control (Fig. 2A). Moreover, the western blot assay showed that transduction of siTUC338 in HCC cells could increase the mRNA and protein levels of RASAL1 (Fig. 2B). These findings provide evidence that TUC338 regulated the expression of RASAL1, regarded as tumor suppressor in many tumors $(14,24)$.

To establish whether siTUC338 plays a suppressing role in HCC tumorigenesis, we analyzed cell viability using MTT assay and invasion ability using Transwell assay in HCC cells transfected with siTUC338. As shown in the left panel of Fig. 2C, knockdown of TUC338 markedly reduced HCC cell viability in HepG2, SMMC7721 and Huh-7 cell lines. Similarly, siTUC338 also significantly decreased incasion ability of human HCC cells (Fig. 2C right panel) in the three cell lines.

siTUC338 activates RASAL1 signaling pathway and inhibits tumor growth genes by directly targeting RASAL1. We next used real-time PCR and western blot to examine whether TUC338 knockdown results in activation of RASAL1 signaling pathway. The immunoblot assay showed an increase 


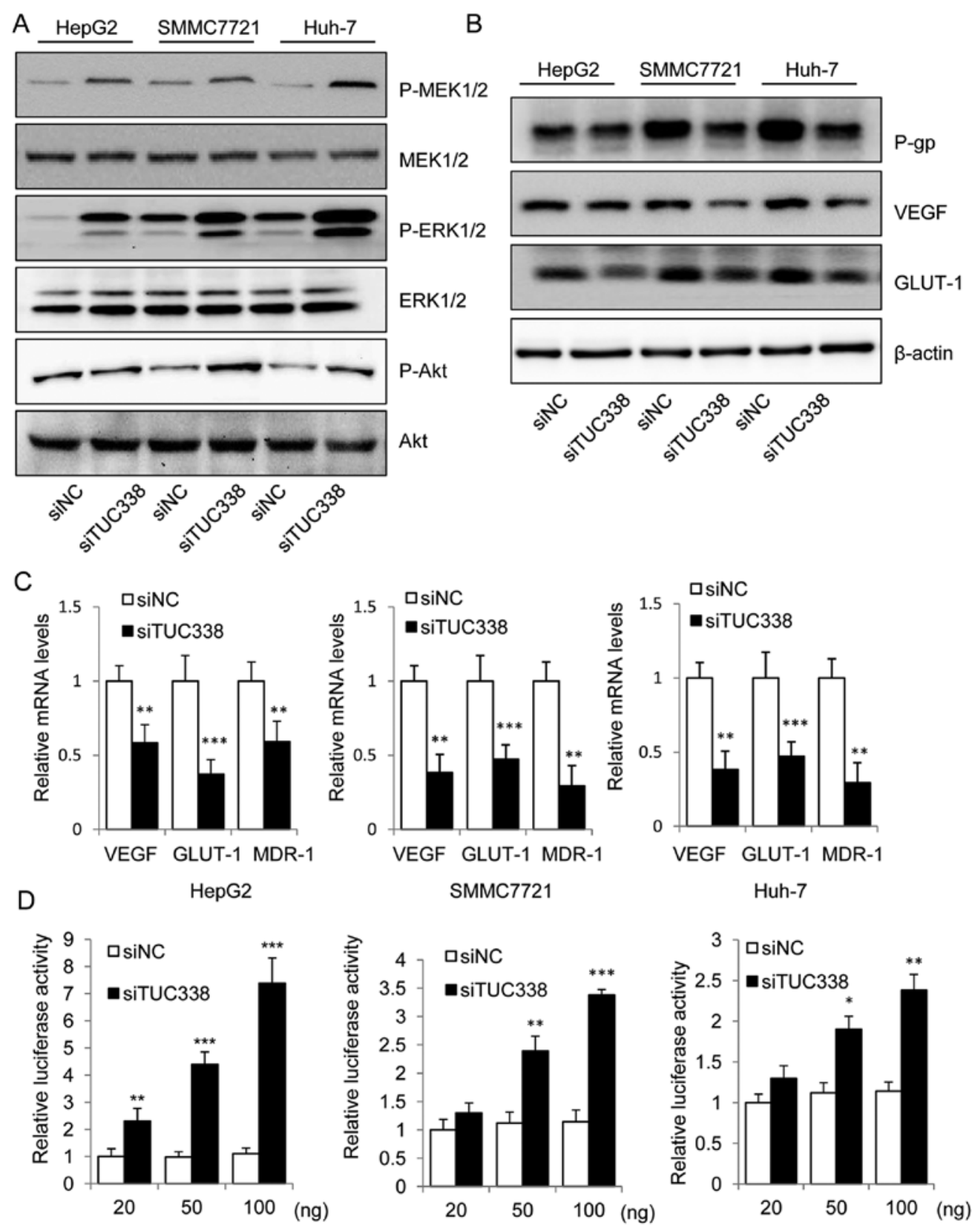

Figure 3. Knockdown TUC338 activates the RASAL1 pathway and inhibits tumor growth genes by directly targeting RASAL1. (A) HepG2, SMMC7721 and Huh-7 cells were transfected with siNC or siTUC338 using Lipo2000, $48 \mathrm{~h}$ later, phosphorylated ERK1/2, MEK1/2 and Akt were assessed by immunoblotting. (B and C) Effect of TUC338 on tumor growth gene expression including VEGF, GLUT-1 and MDR1/P-gp were determined by western blot and real-time PCR. (D) Luciferase assay of HCC cells co-transfected with the luci-RASAL1 reporter with increasing dose of siNC or siTUC 338 . After $48 \mathrm{~h}$, firefly luciferase values were normalized to $\beta$-gal luciferase activity; each bar represents the mean \pm SEM. The results were reproduced in three independent experiments. ${ }^{*} \mathrm{P}<0.05,{ }^{* *} \mathrm{P}<0.01,{ }^{* * * *} \mathrm{P}<0.001$ compared to siNC group. Data are shown as mean $\pm \mathrm{SEM}$ of three independent experiments.

in the phospho-MEK1/2, phospho-ERK1/2 and phospho-Akt in TUC338 knockdown HCC cells (Fig. 3A). Downregulated expression of tumor growth genes vascular endothelial growth factor (VEGF), glucose transporter 1 (GLUT-1), and multidrug resistance gene (MDR1), produces P-glycoprotein; (P-gp) at the transcriptional and the translational levels were also detected after TUC338 knockdown (Fig. 3B and C). Moreover, to determine whether TUC338 could affect the transcriptional activity of RASAL1, we co-transfected RASAL1 luciferase reporter plasmid with siNC or siTUC338 into HepG2, SMMC7721 and Huh-7 cells. Of note, siTUC338 increased the relative luciferase activity in a dose-dependent manner
(Fig. 3D). Collectively, our data demonstrate the functional link between TUC38 and the RASAL1 signaling pathway in human HCC cells.

Knockdown of TUC338 sensitizes HCC cells to sorafenib. The sorafenib resistant subline HepG2/Sor was gengrated by intermittent exposure of sensitive parental HepG2 cells to $30 \mathrm{ng} / \mathrm{ml}$ sorafenib in DMEM culture medium. We treated HepG2, HepG2/Sor and siTUC338 or siNC transfected HepG2/Sor cells with sorafenib and measured cell viability and counted the cell number. Cell viability and cell number results showed that transfected siNC HepG2/Sor cells are highly resistant to 

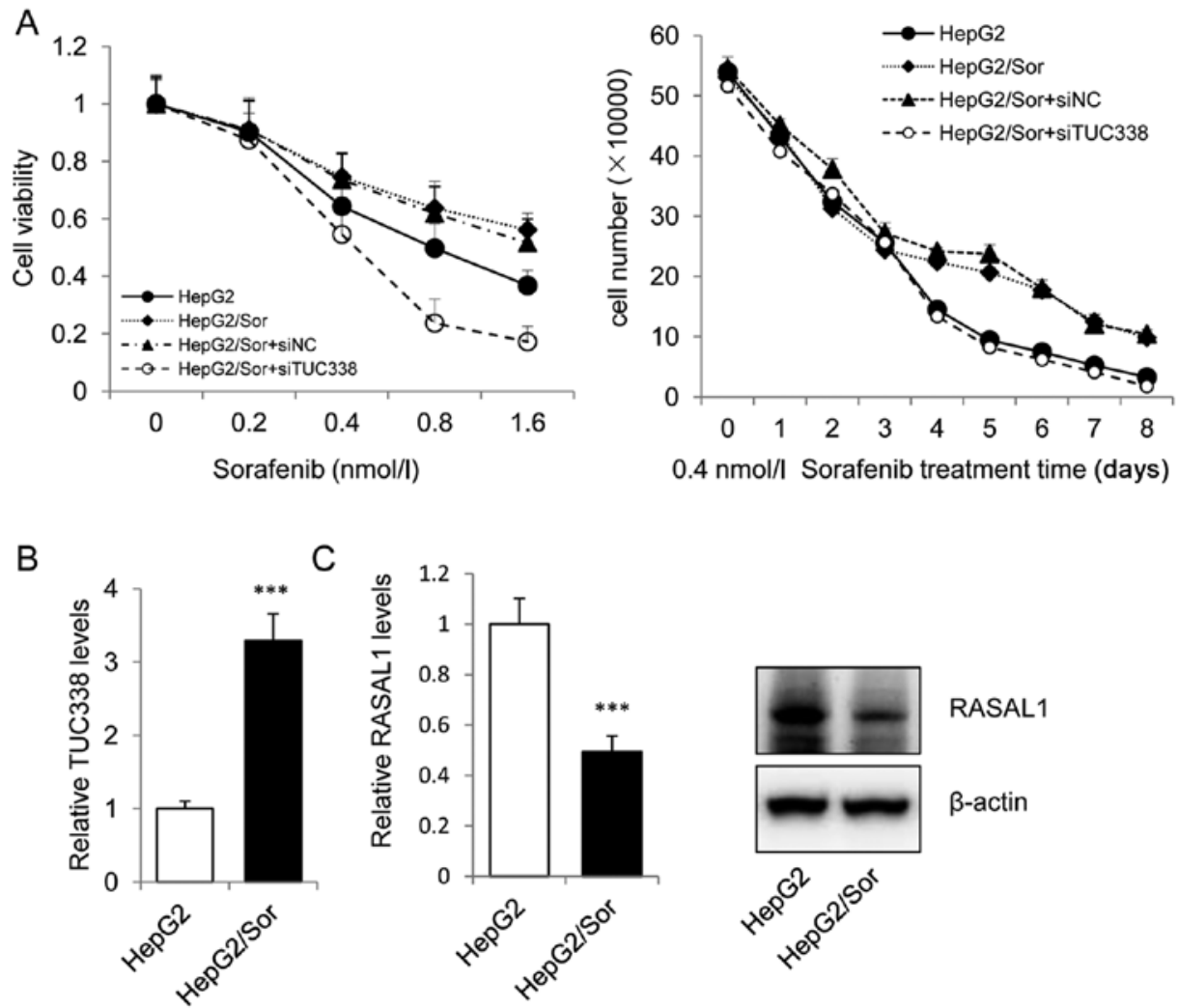

RASAL1

$\beta$-actin

Figure 4. Knockdown of UC338 helps to increase sorafenib treatment response in sorafenib-resistance in vitro. (A) Cell viability is demonstrated as fold change in viability from the negative control in HepG2/Sor cells treated with increasing dose of sorafenib. Cumulative cell growth curve expressed as total cell count of HepG2/Sor cells trasfected with siNC or siTUC338 after treated with $0.4 \mathrm{nM}$ sorafenib over 8 days. (B) Real-time PCR analysis of the relative expression of TUC338 normalized to 18s in HepG2 and HepG2/Sor. (C) Real-time PCR and western blot analysis of RASAL1 protein in HepG2 and HepG2/Sor. Total lysate were prepared either from HepG2 or HepG2/Sor. Antibody against RASAL1 was used in this assay. Protein loading control was done with anti- $\beta$-actin. Each bar represents the mean \pm SEM. The results were reproduced in three independent experiments. ${ }^{* * *} \mathrm{P}<0.001$ compared to HepG2 group. Data are shown as mean \pm SEM of three independent experiments.

sorafenib and that transfection with siTUC338 significantly reduced sorafenib resistance (Fig. 4A). Next, we investigated the mRNA and protein levels of TUC338 and RASAL1 in HepG2/Sor cells using real-time PCR and western blot assay. As shown in Fig. 4B and C, TUC338 was overexpressed and RASAL1 was significantly downregulated both in mRNA and protein levels in HepG2/Sor. Our data revealed that knockdown of TUC338, an lncRNA which was elevated in HCC tissues and cell lines, could sensitizes HCC cells to sorafenib by upregulation of RASAL1, a major tumor suppressor.

Knockdown TUC338 helps to increase sorafenib treatment response in sorafenib-resistant in vivo via increasing the expression of RASAL1. Since lncRNA-TUC338 is capable of regulating RASAL1 expression in vitro, we next used the exnograft tumor nude mouse model to investigate the effect of endogenous TUC338 on RASAL1. After 4 weeks of treatment, the mean tumor volume in the Sor+siTUC338 group was statistically lower compared to that in Sor+siNC group and Sor group (Fig. 5A).

The TUC338 levels in tumor tissues were evaluated by real-time PCR. After intratumoral injection with siTUC338, the TUC338 was significantly decreased in the Sor+siTUC338 group compare with the siNC and physiological saline control group (Fig. 5B).
To understand the functions of TUC338 on the sorafenib treatment by regulating the expression of RASAL1, RASAL1 mRNA and protein levels were valued. Real-time PCR and western blots showed that the expression of RASAL1 both in mRNA and protein levels were upregulated after intratumoral injection with siTUC338 (Fig. 5C).

\section{Discussion}

Resistance to the treatment of sorafenib is one of the major obstacles in current metastatic liver cancer chemotherapy $(25,26)$. Increasing evidence indicates that targeting lncRNA is a promising strategy to improve the efficacy of treatment (27). Although great endeavors have been made, more and more lncRNAs have been discovered as effective chemotherapy inducers or inhibitor, which may be attributed to the complexity of the development of chemotherapy resistance.

To this end, we focused on TUC338, an lncRNA which is overexpressed in liver cancer and may act as a tumor inducer, to illustrate the function of IncRNA in the development process of chemoresistance in liver cancer in vitro and in vivo. Small interfering RNA (siRNA) was used to decrease the expression of TUC338 in HCC cell lines, and its inhibition effect in the proliferation and invasion ability was observed, consistent with previous studies. Noteworthy, the downregulation of 
A
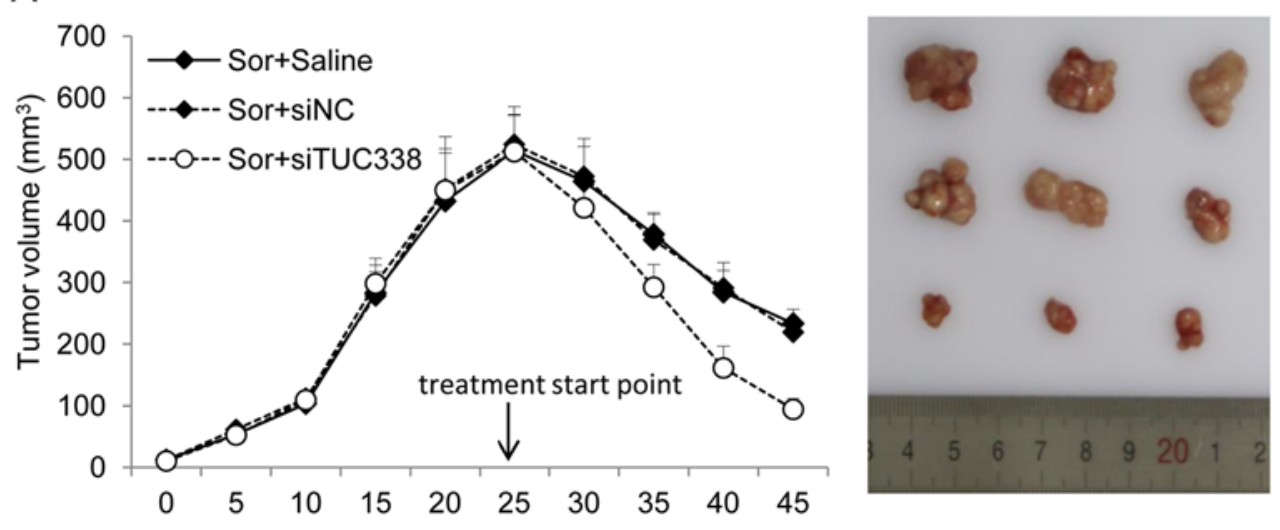

Sor+saline

Sor+siNC

Sor+siTUC338

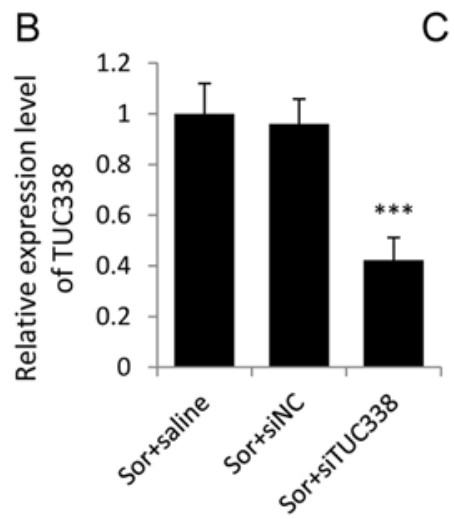

C
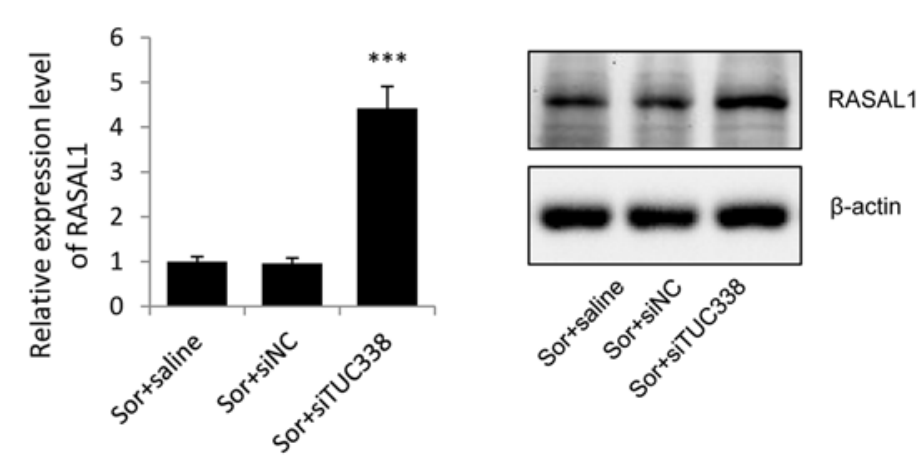

Figure 5. Knockdown of TUC338 helps to increase sorafenib treatment response in sorafenib-resistance in vivo via increasing the expression of RASAL1. (A) Decreased tumor volume in the nude mice after treated with intraperitoneal (i.p.) injection with $4 \mathrm{mg} / \mathrm{kg}$ sorafenib and combination of intratumoral injection of siTUC338 or physiological saline for four weeks. Representative image of HepG2/Sor tumors formed. (B) Expression levels of TUC338 were compared with 18s in HepG2/Sor tumor tissues. (C) The RASAL1 mRNA levels were determined by real-time PCR and RASAL1 protein expression in xenograft tumor tissues were detected by western blot analysis. Each bar represents the mean \pm SEM. The results were reproduced in three independent experiments. ${ }^{* * *} \mathrm{P}<0.001$ compared to Sor + saline group. Data are shown as mean \pm SEM of three independent experiments.

TUC338 was accompanied with the upregulation of RASAL1, the activation of RASAL1 pathway and the inhibition of tumor growth genes and proteins. Luciferase assay showed that siTUC338 increased the relative RASAL1 promoter luciferase activity in a dose-dependent manner, and demonstrated the direct functional link between TUC38 and the RASAL1.

As a tumor suppressor, RASAL1 was found to inhibit cancer progression and as a negative modulator of the RAS signaling pathway by catalyzing RAS inactivation. It was evidenced that enforced rexpression of RASAL1 in gastric cancer cells could suppress cell proliferation and the transformation ability through RAS/ERK signaling pathway $(17,24)$, while activated RAS is involved not only in tumor progression but also possibly in the development of resistance of tumor cells to chemotherapy and ionizing radiation, suggesting targeting this oncogenic protein may be a potential strategy for the treatment of chemoresistance. To this end, a sorafenibresistant liver cancer cell line, HepG2/Sor, was established, which could simplify and better mimic the development process of chemoresistance in liver cancer in vitro. We observed decreased expression of RASAL1 in HepG2/Sor at both mRNA and protein level as compared to that in its parental cell line. More interestingly, this downregulation is concomitant with increased expression of TUC338, which is evidenced as a direct repressor of RASAL1.
Based on in vitro evidence that TUC338 and RASAL1 might be involved in sorafenib-resistance in liver cancer cells, the in vivo efficacy of inhibition of TUC338 in sorafenib treatment were further explored. Our findings that enhanced tumor growth inhibition efficacy induced by sorafenib combined with intratumoral delivering siTUC338 accompanied with overexpression of RASAL1 in tumor xenografts indicates that the TUC338/RASAL1 axis could be a potential therapeutic target for current liver cancer therapy.

The data obtained in the present study suggest that the TUC338/RASAL1 axis might play an essential role in sorafenib-resistance of liver cancer cells, siTUC338 was able to inhibit the RASAL1 pathway and tumor growth genes by directly targeting RASAL1 3'-UTR. Knockdown of TUC338 in HepG2/Sor could sensitize its reaction to the treatment of sorafenib, which was accompanied by the increased expression of RASAL1 both in vivo and in vivo. Suggesting the signaling cohort could serve as a novel therapeutic target for the treatment of chemotherapy resistant liver cancer.

\section{Acknowledgements}

This work was supported by the Natural Science Foundation of China (81201953) and the Natural Science Foundation of Zhejiang Province (Y2090538). 


\section{References}

1. Hernandez-Gea V, Toffanin S, Friedman SL and Llovet JM: Role of the microenvironment in the pathogenesis and treatment of hepatocellular carcinoma. Gastroenterology 144: 512-527, 2013.

2. de Lope CR, Tremosini S, Forner A, Reig M and Bruix J: Management of HCC. J Hepatol 56 (Suppl 1): S75-S87, 2012

3. Spinzi G and Paggi S: Sorafenib in advanced hepatocellular carcinoma. N Engl J Med 359: 2497-2498, 2008.

4. Glackin CA: Targeting the Twist and Wnt signaling pathways in metastatic breast cancer. Maturitas 79: 48-51, 2014.

5. Noguchi K, Katayama K and Sugimoto Y: Human ABC transporter ABCG2/BCRP expression in chemoresistance: Basic and clinical perspectives for molecular cancer therapeutics. Pharm Genomics Pers Med 7: 53-64, 2014.

6. Parasramka MA, Maji S, Matsuda A, Yan IK and Patel T: Long non-coding RNAs as novel targets for therapy in hepatocellular carcinoma. Pharmacol Ther 161: 67-78, 2016.

7. Birney E, Stamatoyannopoulos JA, Dutta A, Guigó R, Gingeras TR, Margulies EH, Weng Z, Snyder M, Dermitzakis ET, Thurman RE, et al; Children's Hospital Oakland Research Institute: Identification and analysis of functional elements in $1 \%$ of the human genome by the ENCODE pilot project. Nature 447: 799-816, 2007.

8. Mercer TR, Dinger ME and Mattick JS: Long non-coding RNAs: Insights into functions. Nat Rev Genet 10: 155-159, 2009.

9. Derrien T, Johnson R, Bussotti G, Tanzer A, Djebali S, Tilgner H, Guernec G, Martin D, Merkel A, Knowles DG, et al: The GENCODE $\mathrm{v} 7$ catalog of human long noncoding RNAs: Analysis of their gene structure, evolution, and expression Genome Res 22: 1775-1789, 2012.

10. Prensner JR and Chinnaiyan AM: The emergence of lncRNAs in cancer biology. Cancer Discov 1: 391-407, 2011.

11. Braconi C, Valeri N, Kogure T, Gasparini P, Huang N, Nuovo GJ, Terracciano L, Croce CM and Patel T: Expression and functional role of a transcribed noncoding RNA with an ultraconserved element in hepatocellular carcinoma. Proc Natl Acad Sci USA 108: 786-791, 2011.

12. Braconi $\mathrm{C}$ and Patel T: Non-coding RNAs as therapeutic targets in hepatocellular cancer. Curr Cancer Drug Targets 12: 1073-1080, 2012.

13. George $\mathrm{J}$ and Patel T: Noncoding RNA as therapeutic targets for hepatocellular carcinoma. Semin Liver Dis 35: 63-74, 2015.

14. Liu D, Yang C, Bojdani E, Murugan AK and Xing M: Identification of RASAL1 as a major tumor suppressor gene in thyroid cancer. J Natl Cancer Inst 105: 1617-1627, 2013.

15. Ohta M, Seto M, Ijichi H, Miyabayashi K, Kudo Y, Mohri D, Asaoka Y, Tada M, Tanaka Y, Ikenoue T, et al: Decreased expression of the RAS-GTPase activating protein RASAL1 is associated with colorectal tumor progression. Gastroenterology 136: 206-216, 2009.
16. Ngeow $\mathbf{J}$ and Eng C: RASAL1 in thyroid cancer: Wisdom from an old foe. J Natl Cancer Inst 105: 1597-1599, 2013.

17. Chen H, Cheng Z-Y, Pan Y, Wang Z, Liu Y and Zhang J-Q RASAL1 influences the proliferation and invasion of gastric cancer cells by regulating the RAS/ERK signaling pathway. Hum Cell 27: 103-110, 2014.

18. Kolfschoten IG, van Leeuwen B, Berns K, Mullenders J, Beijersbergen RL, Bernards R, Voorhoeve PM and Agami R: A genetic screen identifies PITX1 as a suppressor of RAS activity and tumorigenicity. Cell 121: 849-858, 2005.

19. Walker SA, Kupzig S, Bouyoucef D, Davies LC, Tsuboi T, Bivona TG, Cozier GE, Lockyer PJ, Buckler A, Rutter GA, et al: Identification of a Ras GTPase-activating protein regulated by receptor-mediated $\mathrm{Ca}^{2+}$ oscillations. EMBO J 23: 1749-1760, 2004.

20. Loriot Y, Mordant P, Deutsch E, Olaussen KA and Soria J-C: Are RAS mutations predictive markers of resistance to standard chemotherapy? Nat Rev Clin Oncol 6: 528-534, 2009.

21. Li W, Zhai B, Zhi H, Li Y, Jia L, Ding C, Zhang B and You W: Association of ABCB1, $\beta$ tubulin I, and III with multidrug resistance of MCF7/DOC subline from breast cancer cell line MCF7. Tumour Biol 35: 8883-8891, 2014.

22. Febriansah R, Putri DD, Sarmoko, Nurulita NA, Meiyanto E and Nugroho AE: Hesperidin as a preventive resistance agent in MCF-7 breast cancer cells line resistance to doxorubicin. Asian Pac J Trop Biomed 4: 228-233, 2014.

23. Tomayko MM and Reynolds CP: Determination of subcutaneous tumor size in athymic (nude) mice. Cancer Chemother Pharmacol 24: 148-154, 1989 .

24. Qiao F, Su X, Qiu X, Qian D, Peng X, Chen H, Zhao Z and Fan H Enforced expression of RASAL1 suppresses cell proliferation and the transformation ability of gastric cancer cells. Oncol Rep 28: 1475-1481, 2012.

25. Villanueva A and Llovet JM: Second-line therapies in hepatocellular carcinoma: Emergence of resistance to sorafenib. Clin Cancer Res 18: 1824-1826, 2012.

26. Zhai B and Sun X-Y: Mechanisms of resistance to sorafenib and the corresponding strategies in hepatocellular carcinoma. World J Hepatol 5: 345-352, 2013.

27. Yu W, Qiao Y, Tang X, Ma L, Wang Y, Zhang X, Weng W, Pan Q, $\mathrm{Yu}$ Y, Sun F, et al: Tumor suppressor long non-coding RNA, MT1DP is negatively regulated by YAP and Runx2 to inhibit FoxA1 in liver cancer cells. Cell Signal 26: 2961-2968, 2014. 